\title{
Medicação potencialmente inapropriada em idosos acompanhados em cuidados domiciliários nos últimos 12 meses de vida
}

Marlene Areias, ${ }^{1}$ Paulo Reis-Pina ${ }^{2-4}$

\section{RESUMO}

Introdução: A medicação potencialmente inapropriada (MPI) é cada vez mais alvo de estudo e atenção, pelas consequências negativas que pode ter num doente vulnerável.

Objetivo: Determinar a prevalência da MPI e das interações medicamentosas em idosos acompanhados por equipas domiciliárias nos últimos doze meses de vida.

Material e Métodos: Estudo quantitativo, descritivo, retrospetivo e observacional. Identificação da MPI, usando os critérios STOPPFrail, nos idosos com $\geq 65$ anos acompanhados pelas equipas comunitárias de suporte em cuidados paliativos nos Açores, que faleceram entre janeiro de 2016 e dezembro de 2018.

Resultados: Foram incluídos 137 doentes, sendo 72 homens (52,55\%), com uma mediana de 78 anos (amplitude interquartil 73-84). A prevalência de MPI foi de 90,51\% ( $n=124)$, existindo sobretudo prescrição de medicação sem evidência clínica clara $(51,09 \%, n=70)$ e de inibidores da bomba de protões $(45,99 \%, n=63)$. Foram contabilizadas 1.107 interações medicamentosas em 120 doentes (87,59\%). A MPI esteve envolvida nas interações de 98 doentes $(71,53 \%)$ e em 473 das interações medicamentosas clinicamente significativas $(46,78 \%)$.

Conclusão: A prevalência de MPI é elevada em idosos nos últimos doze meses de vida. O uso dos critérios STOPPFrail pode ajudar a identificar a MPI e a delinear um plano para a sua redução, de preferência num contexto de trabalho interdisciplinar.

Palavras-chave: Cuidados paliativos; Efeitos colaterais e reações adversas relacionados com medicamentos; Idoso; Lista de medicamentos potencialmente inapropriados; Polifarmácia.

\section{INTRODUÇÃO}

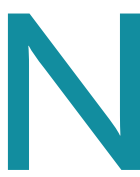

os países desenvolvidos, o aumento da esperança média de vida tem sido acompanhado por um incremento das doenças limitadoras da vida (cancro, insuficiências de órgão, doenças neurodegenerativas, etc.) e das doenças crónicas, como a hipertensão arterial, a diabetes mel-

1. Unidade de Saúde da Itha Terceira. Angra do Heroísmo, Açores, Portugal.

2. Unidade de Cuidados Paliativos Bento Menni, Casa de Saúde da Idanha. Sintra, Portugal.

3. Escola de Medicina da Universidade do Minho. Braga, Portugal.

4. Faculdade de Medicina da Universidade de Lisboa. Lisboa, Portugal. litus, a dislipidemia, as perturbações da ansiedade e da depressão, entre outras. Todas estas doenças crónicas, progressivas e/ou incuráveis, isoladamente ou em associação, cursam com complexidade, ${ }^{1-4}$ polimedicação e sobrecarga terapêutica. ${ }^{5}$

Nos indivíduos com doenças crónicas é possível haver prescrição, por um lado, de medicamentos para tratar e/ou prevenir exacerbações e comorbilidades; por outro, de medicamentos para controlar os sintomas associados à patologia e à progressão clínica. ${ }^{6-7}$ Neste contexto, a polimedicação é um cenário frequente, existindo um aumento de medicação potencialmente inapropriada (MPI) e outras consequências negativas..$^{5,8-10}$ 
A MPI engloba a medicação que é prescrita: i) sem indicação clínica ou com indicação errada; ii) por períodos demasiado curtos ou longos; iii) em doses incorretas; iv) com um elevado risco de reações adversas medicamentosas (RAM), incluindo interações medicamentosas (IMED); v) com custo acrescido para o serviço de saúde. ${ }^{1-3}$

Nas doenças avançadas e irreversíveis também podem considerar-se MPI os fármacos utilizados para prevenção primária, secundária ou aqueles sem benefício a curto prazo, uma vez que a esperança de vida de muitos doentes é limitada, mormente se for considerado o tempo necessário para existir benefício clínico e a referenciação tardia para as equipas de cuidados paliati$\operatorname{vos}(\mathrm{CP}){ }^{1}$

Para a deteção da MPI existem várias ferramentas disponíveis, sendo a STOPPFrail (Screening Tool of Older Persons Prescriptions in Frail adults with limited life expectancy) uma das mais recentes. Esta ferramenta congrega 27 critérios que foram desenvolvidos com base na necessidade de identificar a MPI num grupo de pessoas com complexidade crescente. ${ }^{11-12}$

São escassos os estudos que abordam a prevalência da MPI nos doentes seguidos na comunidade por equipas de CP, sendo raramente utilizada a STOPPFrail. É relevante aprofundar o conhecimento sobre esta realidade no contexto comunitário para que se possam desenvolver estratégias de redução da MPI e das suas potenciais consequências negativas.

O objetivo deste estudo foi determinar a prevalência da MPI e o número de IMED em idosos, acompanhados por equipas domiciliárias de $\mathrm{CP}$, durante os últimos doze meses de vida.

\section{MATERIAL E MÉTODOS}

Estudo quantitativo, descritivo, observacional e retrospetivo. A amostra foi de conveniência, constituída por doentes com $\geq 65$ anos que faleceram entre 1 de janeiro de 2016 e 31 de dezembro de 2018 e que eram acompanhados por uma Equipa Comunitária de Suporte em Cuidados Paliativos da Região Autónoma dos Açores.

Foram registados dados individuais relativos a: idade, género, lista de problemas de saúde, medicação prescrita nos últimos doze meses de vida. Foi usada a classificação internacional das doenças do ICD-10-CM. ${ }^{13}$
Todos os medicamentos foram agrupados segundo a classificação farmacocinética que consta do Prontuário Terapêutico da Autoridade Nacional do Medicamento e Produtos de Saúde, vulgo Infarmed ${ }^{\circledR} .^{14}$

Para identificar a MPI foram utilizados os 27 critérios da STOPPFrail..$^{11-12}$ Para identificar as IMED foi usado o programa Lexi-interact $\AA_{(L e x i-C o m p} \odot$, Inc., Hudson, Ohio, EUA). Segundo este, existem cinco níveis de IMED: os níveis X, D e C dizem respeito a IMED que poderão ser clinicamente significativas, ditas «potenciais»; o nível B congrega as IMED sem impacto clínico, não sendo necessária qualquer ação clínica; o nível A significa que não há IMED conhecidas.

A análise descritiva dos dados foi feita com o programa IBM SPSS Statistics ${ }^{\circledR}$ v. 26.0 (IBM Corporation@), Armonk, NY, EUA). Os dados qualitativos foram apresentados com frequências e percentagens; os dados quantitativos com medianas e amplitudes interquartis (AIQ).

O estudo foi aprovado pela Comissão de Ética do Centro Académico de Medicina de Lisboa e pela Comissão de Ética da Unidade de Saúde da Ilha Terceira.

\section{RESULTADOS}

Resultados gerais

A amostra foi de 137 doentes, sendo 72 homens $(52,55 \%)$ com idade mediana de 78 anos. A maioria $(91,97 \%)$ dos doentes teve um acompanhamento de $<12$ meses, com uma mediana de dois meses (Tabela $1)$.

Foram classificados 591 problemas de saúde, com mediana de quatro por doente (AIQ 3-6), 217 doenças limitadoras de vida e 374 comorbilidades. Das doenças limitadoras de vida, 58,06\% ( $n=126)$ foram neoplasias, sobretudo do sistema digestivo $(35,71 \%, n=45)$. A maioria das comorbilidades foi do sistema circulatório $(86,13 \%, n=118)$ e doenças endócrinas $(81,75 \%, n=112)$.

Foram prescritos 1.127 fármacos, com uma mediana de oito fármacos/doente (AIQ 5-10,5). A maioria dos doentes $(80,29 \%)$ teve prescritos $\geq$ cinco medicamentos (Figura 1). Os medicamentos mais prescritos foram: opioides (48,18\%, $n=66)$, ansiolíticos $(48,18 \%, n=66) \mathrm{e}$ inibidores da bomba de protões (IBP) $(45,98 \%, n=63)$.

Do total de medicamentos prescritos não se encontrou evidência clínica clara para 131 prescrições (11,62\%). 


\begin{tabular}{|l|c|c|}
\hline \multicolumn{2}{|c|}{ TABELA 1. Caracterização da amostra $(n=137)$} \\
\hline Doentes & $\mathbf{n}(\%)$ & Mediana (AIQ) \\
\hline Género feminino & $137(100)$ & - \\
\hline Género masculino & $65(47,45)$ & - \\
\hline Idade (anos) & - & - \\
\hline Tempo de seguimento & & $78(73-84)$ \\
\hline $\begin{array}{l}\text { Ano } \\
\text { Meses }\end{array}$ & 0 & 0 \\
Dias & $126(91,97 \%)$ & $2(0-4)$ \\
\hline
\end{tabular}

Legenda: $\mathrm{AIQ}=$ Amplitude interquartil. tares $(4,84 \%, n=23$, sendo todos vitamínicos) (Tabela 2).

Dos medicamentos que os doentes não conseguiram tomar ou tolerar (critério A1) destacam-se: ansiolíticos, sedativos e hipnóticos $(17,46 \%, n=11)$; antidepressivos $(9,52 \%, n=6)$ e opioides $(7,94 \%, n=5)$. Dos medicamentos sem evidência clínica clara (critério A2), os mais frequentes foram: antianginosos, anticonvulsivantes, compostos de ferro, dopaminomiméticos, antigotosos e vasodilatadores (Tabela 3 ).

Interações medicamentosas

Houve 1.107 IMED em 120 doentes (87,59\%). Em 117 doentes $(85,40 \%)$ foram registadas 1.011 potenciais IMED (91,33\%). Apenas três doentes tiveram IMED sem impacto clínico (nível B). A maioria das IMED foi de nível C $(70,91 \%)$ e de nível D (18,52\%), existindo em 112 doentes $(81,75 \%)$ e 74 doentes $(54,01 \%)$, respetivamente. A IMED potencial mais rara foi do nível X (1,90\%) em 14 doentes $(10,22 \%)$ (Tabela 4$)$.

A MPI esteve envolvida nas IMED de 98 doentes $(71,53 \%)$ e em 473 de todas as potenciais IMED $(46,79 \%)$, sendo 371 do nível C, 90 do nível D e 12 do nível X. Dos 117 doentes

\section{Medicação potencialmente inapropriada}

A prescrição de MPI envolveu 475 fármacos e ocorreu em 124 idosos (90,51\%). Destes, 101 (81,45\%) reuniram $\geq$ dois critérios (mediana de quatro critérios, AIQ 1-5). Os critérios mais identificados foram o «A2: Medicamento sem evidência clínica clara» em 70 doentes $(51,09 \%)$ e o «E1: Inibidores da Bomba de Protões» em 63 doentes (45,99\%). Dos 27 critérios, 13 não foram reportados (Tabela 2).

Metade da MPI foi representada por: IBP $(13,26 \%$, $n=63)$, antidiabéticos orais $(10,11 \%, n=48)$, antipsicóti$\cos (9,89 \%, n=47)$, antidislipidémicos $(7,37 \%, n=35$, sendo 31 estatinas), antiagregantes plaquetários na prevenção primária $(4,84 \%, n=23)$ e suplementos alimen- com potenciais IMED, a MPI mais frequente esteve representada por medicação sem evidência clínica clara (critério A2) em 45 doentes $(38,46 \%)$ e antipsicóticos (critério D1) em 38 doentes (32,48\%).

Os medicamentos sem evidência clínica clara (critério A2) foram encontrados em 122 das potenciais IMED $(12,07 \%)$ sendo que, em aproximadamente um terço destes casos, as classes farmacológicas mais frequentes foram: antianginosos, anticonvulsivantes, antiarrítmicos e dopaminomiméticos (Tabela 5).

\section{DISCUSSÃO}

Nos últimos doze meses de vida de idosos seguidos por equipas de CP verificou-se que em cada dez doentes: nove tiveram MPI, cinco tiveram prescrições sem 


\begin{tabular}{|c|c|c|}
\hline Critérios & $\begin{array}{c}\text { Doentes }(n=137) \\
n(\%)\end{array}$ & $\begin{array}{c}\text { MPI }(n=475) \\
n(\%)\end{array}$ \\
\hline \multicolumn{3}{|l|}{ Secção A: Geral } \\
\hline 1. Medicamento que o doente não consegue tomar ou tolerar & $17(12,41)$ & $63(13,26)$ \\
\hline 2. Medicamento sem evidência clínica clara & $70(51,09)$ & $131(27,58)$ \\
\hline \multicolumn{3}{|l|}{ Secção B: Cardiovascular } \\
\hline 1. Antidislipidémicos & $32(23,36)$ & $35(7,37)$ \\
\hline 2. Alfa-bloqueantes para o tratamento da HTA & 0 & 0 \\
\hline \multicolumn{3}{|l|}{ Secção C: Coagulação } \\
\hline 1. Antiagregantes plaquetários na prevenção primária & $23(16,79)$ & $23(4,84)$ \\
\hline \multicolumn{3}{|l|}{ Secção D: Sistema nervoso central } \\
\hline 1. Antipsicóticos & $39(28,47)$ & $47(9,89)$ \\
\hline 2. Memantina & $5(3,65)$ & $5(1,05)$ \\
\hline \multicolumn{3}{|l|}{ Secção E: Gastrointestinal } \\
\hline 1. Inibidores da bomba de protões & $63(45,99)$ & $63(13,26)$ \\
\hline 2. Antagonistas dos recetores $\mathrm{H} 2$ da histamina & 0 & 0 \\
\hline 3. Antiespasmódicos & 0 & 0 \\
\hline \multicolumn{3}{|l|}{ Secção F: Respiratório } \\
\hline 1. Teofilina & 0 & 0 \\
\hline 2. Antagonista dos leucotrienos & 0 & 0 \\
\hline \multicolumn{3}{|l|}{ Secção G: Músculo-esquelético } \\
\hline 1. Suplemento de cálcio & $1(0,73)$ & $1(0,21)$ \\
\hline 2. Medicamentos que atuam no osso e no metabolismo do cálcio & 0 & 0 \\
\hline 3. Modulador seletivo dos recetores de estrogénio na osteoporose & 0 & 0 \\
\hline 4. AINE oral por períodos longos & $7(5,11)$ & $7(1,47)$ \\
\hline 5. Glucocorticoide oral por longos períodos de tempo & $15(10,95)$ & $15(3,16)$ \\
\hline \multicolumn{3}{|l|}{ Secção H: Genito-urinário } \\
\hline 1. Inibidores da alfa 5 -redutase & 0 & 0 \\
\hline 2. Alfa-bloqueantes & 0 & 0 \\
\hline 3. Antagonistas muscarínicos & 0 & 0 \\
\hline \multicolumn{3}{|l|}{ Secção I: Sistema endócrino } \\
\hline 1. Antidiabético oral & $29(21,17)$ & $48(10,11)$ \\
\hline 2. IECA na diabetes & 0 & 0 \\
\hline 3. Antagonista dos recetores da angiotensina na diabetes & 0 & 0 \\
\hline 4. Estrogénio sistémico nos sintomas da menopausa & 0 & 0 \\
\hline \multicolumn{3}{|l|}{ Secção J: Miscelânea } \\
\hline 1. Suplemento alimentar & $18(13,14)$ & $23(4,84)$ \\
\hline 2. Suplemento nutricional & $5(3,65)$ & $6(1,26)$ \\
\hline 3. Antibiótico profilático & $7(5,11)$ & $8(1,68)$ \\
\hline
\end{tabular}

Legenda: HTA = Hipertensão arterial; AINE = Anti-inflamatório não esteroide; IECA = Inibidor da enzima de conversão da angiotensina 
TABELA 3. Classes farmacológicas da medicação potencialmente inapropriada (critérios A1 e A2) ( $n=194)$

\begin{tabular}{|c|c|c|c|}
\hline $\begin{array}{l}\text { A1. Medicamento que o doente não consegue } \\
\text { tomar ou tolerar }\end{array}$ & $n(\%)$ & A2. Medicamento sem evidência clínica clara & $n(\%)$ \\
\hline Ansiolítico, sedativos e hipnóticos & $11(17,46)$ & Antianginoso & $15(11,45)$ \\
\hline Antidepressivo & $6(9,52)$ & Anticonvulsivante & $7(5,34)$ \\
\hline Opioide & $5(7,94)$ & Composto de ferro & $7(5,34)$ \\
\hline Analgésico & $4(6,35)$ & Dopaminomimético & $7(5,34)$ \\
\hline Antiemético & $4(6,35)$ & Medicamento usado no tratamento da gota & $7(5,34)$ \\
\hline Bloqueador dos canais de cálcio & $3(4,76)$ & Vasodilatador & $7(5,34)$ \\
\hline Diurético da ansa & $3(4,76)$ & Venotrópico & $6(4,58)$ \\
\hline Dopaminomimético & $3(4,76)$ & Xantina & $7(4,58)$ \\
\hline Laxante de contacto & $3(4,76)$ & Medicamento usado na retenção urinária & $5(3,82)$ \\
\hline Inibidor da enzima de conversão da angiotensina & $3(4,76)$ & $\begin{array}{l}\text { Medicamento usado no tratamento da anemia } \\
\text { megaloblástica }\end{array}$ & $5(3,82)$ \\
\hline Anticonvulsivante & $2(3,17)$ & Beta-bloqueante & $4(3,05)$ \\
\hline Antipsicótico & $2(3,17)$ & Diurético da ansa & $4(3,05)$ \\
\hline Outro $(n=1)$ & $14(22,22)$ & Heparina & $4(3,05)$ \\
\hline & & Inibidor da enzima de conversão da angiotensina & $4(3,05)$ \\
\hline & & Antiagregante & $3(2,29)$ \\
\hline & & Antiarrítmico & $4(2,29)$ \\
\hline & & Anti-histamínico & $5(2,29)$ \\
\hline & & Antivertiginoso & $6(2,29)$ \\
\hline & & Bloqueador dos canais de cálcio & $7(2,29)$ \\
\hline & & Outros ( $n=1$ ou $n=2)$ & $28(21,37)$ \\
\hline
\end{tabular}

evidência clínica clara, quatro tomaram um IBP, oito tiveram IMED. A MPI esteve envolvida nas IMED de sete em cada dez doentes. A MPI representou quase metade das IMED clinicamente significativas.

\section{Medicação potencialmente inapropriada}

A prevalência de $90,51 \%$ da MPI foi bastante superior à encontrada noutros estudos - de $16 \%$ a 88,5\% realizados no âmbito dos CP, quer na comunidade quer no hospital. ${ }^{8,10,12,15-22}$ Estas variações devem-se aos diferentes métodos usados nas investigações, mormente em relação a: seleção dos doentes; tipo de doença em causa; tipologia dos cuidados (primário, secundário ou terciário); critérios para identificar a MPI; momento da trajetória da doença; seguimento e tratamento dos doentes por diferentes profissionais de saúde; tempo de seguimento em CP; expectativas e preferências dos doentes; entre outros métodos.

A ferramenta STOPPFrail, além de apoiar a prescrição de medicamentos em doentes com esperança de vida limitada, é fácil de usar, de tamanho curto, organizada por sistemas, eficiente em termos de tempo e, portanto, mais viável de implementar na prática clínica diária. ${ }^{23}$ Estudos recentes que usaram a STOPPFrail mostraram uma prevalência de MPI de $67 \%$ a $80 \%$, o que pode indicar uma boa adaptação da ferramenta aos doentes em CP e suas medicações. ${ }^{12}$ A STOPPFrail, como outras ferramentas, deve funcionar como guia, quer no momento de início quer de continuação da medicação, não devendo excluir, nem substituir, uma avaliação clínica global, que é essencial para determinar a MPI. 
TABELA 4. Distribuição dos 120 doentes que registaram interações medicamentosas (Parte A) e distribuição da globalidade das 1.107 interações medicamentosas (Parte B)

Níveis de interações e suas combinações

Parte A

\begin{tabular}{|c|c|c|c|c|c|c|c|c|c|c|c|c|c|c|c|c|}
\hline \multirow{4}{*}{$\begin{array}{l}\text { Níveis de } \\
\text { interações } \\
\text { (\#) }\end{array}$} & $X$ & 0 & 1 & 3 & 0 & 4 & 3 & 0 & 3 & $(*)$ & $(*)$ & $(*)$ & $(*)$ & $(*)$ & $(*)$ & 14 \\
\hline & D & 1 & 3 & 31 & 1 & $\left({ }^{*}\right)$ & $(*)$ & $(*)$ & 31 & 4 & 0 & 3 & $(*)$ & $(*)$ & $\left({ }^{*}\right)$ & 74 \\
\hline & C & 3 & 31 & 24 & 13 & $(*)$ & $(*)$ & 31 & $(*)$ & $(*)$ & 3 & $(*)$ & 4 & 3 & $(*)$ & 112 \\
\hline & B & 0 & 1 & 13 & 3 & $(*)$ & 31 & $(*)$ & $\left({ }^{*}\right)$ & $(*)$ & $(*)$ & 3 & $(*)$ & 0 & 4 & 55 \\
\hline
\end{tabular}

Parte B

\begin{tabular}{|c|c|c|c|c|c|c|c|c|c|c|c|c|c|c|c|c|}
\hline \multirow{4}{*}{$\begin{array}{l}\text { Níveis de } \\
\text { interações } \\
\text { (\#) }\end{array}$} & $x$ & 0 & 2 & 3 & 0 & 8 & 3 & 0 & 5 & 0 & 0 & 0 & 0 & 0 & 0 & 21 \\
\hline & D & 3 & 3 & 72 & 3 & 0 & 0 & 0 & 78 & 27 & 0 & 19 & 0 & 0 & 0 & 205 \\
\hline & C & 12 & 158 & 96 & 109 & 0 & 0 & 293 & 0 & 0 & 29 & 0 & 54 & 34 & 0 & 785 \\
\hline & B & 0 & 2 & 16 & 3 & 0 & 54 & 0 & 0 & 0 & 0 & 6 & 0 & 0 & 15 & 96 \\
\hline
\end{tabular}

Legenda: (\#) Não houve nenhuma interação do nível A; $\left(^{*}\right)$ Combinação já assinalada noutra célula.

\begin{tabular}{|c|c|c|c|}
\hline Classe farmacológica & $n$ & Classe farmacológica & $n$ \\
\hline Antianginoso & 12 & $\begin{array}{l}\text { Medicamento utilizado no tratamento sintomático das alterações } \\
\text { das funções cognitivas }\end{array}$ & 4 \\
\hline Antiarrítmico & 11 & Antagonista colinérgico & 3 \\
\hline Anticonvulsivante & 10 & Digoxina & 3 \\
\hline Dopaminomimético & 10 & Medicamento usado na retenção urinária & 3 \\
\hline Medicamento usados para o tratamento da gota & 8 & Bloqueador dos canais de cálcio & 2 \\
\hline Xantinas & 8 & Glucocorticoide inalado & 2 \\
\hline Anti-histamínico & 7 & Outro antidiabético & 2 \\
\hline Vasodilatador & 7 & Anticoagulante & 1 \\
\hline Antiagregante & 6 & Antivertiginoso & 1 \\
\hline Diurético da ansa & 6 & Antagonista receptores da angiotensina & 1 \\
\hline Heparina & 5 & Bifosfonato & 1 \\
\hline Beta-bloqueante & 4 & Medicamento para tratamento da litíase biliar & 1 \\
\hline Inibidor da enzima de conversão da angiotensina & 4 & & \\
\hline
\end{tabular}

(critério A2) nas interações medicamentosas clinicamente significativas ( $n=122)$

Têm sido associados ao aumento da MPI alguns elementos clínicos e sociodemográficos, nomeadamente: idade avançada, género feminino, insónia, perturbações da ansiedade, síndroma depressivo, quedas, dor e deterioração cognitiva. ${ }^{16}$ No entanto, o uso do STOPPFrail não revelou diferenças de MPI em função de: género, síndromas geriátricos, comorbilidades e tipo de doença que necessita de CP. ${ }^{18}$ 
A maioria dos estudos, abordando a MPI em CP, foi realizada em contexto hospitalar. O internamento hospitalar está normalmente associado a uma agudização do estado geral, pelo que é de esperar que haja um aumento de medicação para controlo sintomático, sendo assim explicadas as elevadas prevalências de MPI, de $80 \%$ a $88 \%{ }^{12,16,19}$ São necessários mais estudos que envolvam outros contextos para além do hospitalar.

Um dos fatores de risco para a MPI é a polimedicação. ${ }^{18,24}$ Esta é geralmente abordada numa perspetiva numérica, sem ter em conta as classes farmacológicas envolvidas, os estados da doença e as comorbilidades. Isto dificulta a avaliação da segurança e adequação da medicação ao contexto clínico, condicionando a comparação da polimedicação apropriada versus inapropriada. ${ }^{24}$ Por conseguinte, a polimedicação deve ser vista de modo crítico, podendo ser apropriada nos idosos com multimorbilidade sempre que: 1) seja aplicada uma abordagem holística no ato da prescrição; 2) o uso da medicação seja otimizado e a medicação prescrita de acordo com a melhor evidência; 3 ) seja questionando o benefício pretendido com o tratamento, com o objetivo de melhorar a qualidade de vida. ${ }^{25-28}$

Nas doenças crónicas, à medida que ocorre a progressão clínica e se aproxima o fim de vida (FDV), a prioridade médica deve concentrar-se no controlo do sofrimento evitável e na garantia da melhor qualidade de vida. Este facto pode explicar o aumento do número de medicamentos que se observa no FDV, em que a taxa de doentes a tomar $\geq 10$ medicamentos/dia passa de $30,2 \%$ para $47,2 \%$ no último mês de vida. ${ }^{29}$ Este aumento observa-se em várias classes farmacológicas, muitas delas fundamentais nos serviços de CP, como antipsicóticos, ansiolíticos, opioides, glucocorticoides, antieméticos e laxantes. ${ }^{17,29-32}$ Neste estudo, as classes farmacológicas mais prescritas foram: ansiolíticos $(48,18 \%)$, opioides $(48,18 \%)$ e IBP $(45,98 \%)$. Os opioides corresponderam a $8,96 \%$ de toda a prescrição $(n=101)$ e os ansiolíticos a 7,01\% ( $n=79)$.

A elevada prevalência de MPI documentada teve como contribuintes a medicação preventiva e aquela destinada a tratar as comorbilidades. Nas doenças crónicas em fases avançadas, quando a situação é considerada irreversível, e privilegiando o adequado controlo sintomático, deve existir uma diminuição dos fármacos modificadores da doença incurável e dos fár- macos com intuito preventivo. Todavia, tal nem sempre sucede, podendo estes fármacos significar $25 \%$ da medicação dos doentes em FDV. ${ }^{12}$ A literatura indica que as classes farmacológicas mais presentes na MPI são: estatinas, antiagregantes, anti-hipertensores, vasodilatadores, antidiabéticos orais, IBP, suplementos minerais e vitamínicos, medicamentos usados no tratamento da osteoporose e gota. ${ }^{2,29,31,33-35}$ Neste estudo confirmou-se que as classes farmacológicas mais frequentes no tratamento preventivo e/ou das comorbilidades, e causadoras de MPI, foram: IBP em 45,99\% ( $n=63)$, estatinas em 22,63\% ( $n=31)$, antidiabéticos orais em $21,17 \%(n=29)$, antiagregantes em 19,71\% $(n=27) \mathrm{e}$ suplementos alimentares em $13,14 \%$ dos casos $(n=18)$. Pese embora estes dados, é sabido que existem alguns medicamentos que permitem modificar a doença, controlar as comorbilidades e assegurar o controlo sintomático, como os beta-bloqueantes (uso nas palpitações) e os antidiabéticos (uso na hiperglicemia sintomática). Destarte, é pertinente aclarar a indicação de cada medicamento do idoso, de forma a avaliar corretamente a sua adequação, evitando precocemente a MPI e as IMED.

\section{Interações medicamentosas}

As IMED podem surgir associadas à MPI, podendo contribuir para: declínio funcional e cognitivo, redução da qualidade e do tempo de vida, sobrecarga terapêutica, idas recorrentes ao serviço de urgência, hospitalização não programada e aumento dos custos em saúde. ${ }^{2,8-9,911,36}$ Neste estudo a prevalência de IMED foi de $85,40 \%(n=117)$, bastante superior à encontrada na literatura que varia de $2,69 \%$ a $75 \%$ no âmbito dos $\mathrm{CP}^{37-42}$ O risco de IMED aumenta com o número de medicamentos, sendo de $83,30 \%$ em doentes a fazer $\geq 11$ medicamentos. ${ }^{42}$

Constatou-se que $46,78 \%$ das IMED podiam considerar-se evitáveis. Na medicação crónica as classes farmacológicas consideradas como MPI, e mais frequentemente envolvidas nas IMED, são: anticoagulantes, insulina, antagonistas da dopamina e anticolinérgi$\cos ^{37,40,43}$ Neste estudo foram os antianginosos, os antiarrítmicos, os anticonvulsivantes e os dopaminomiméticos.

O conhecimento e a compreensão dos medicamentos mais associados às IMED, os seus mecanismos de 
ação e os sintomas secundários podem ajudar os médicos na escolha idónea dos fármacos, na identificação precoce das IMED, na monitorização e ajuste da medicação, principalmente dos princípios ativos mais usados em CP, como a metoclopramida e o haloperidol. ${ }^{40}$

A presença de MPI, de acordo com a STOPPFrail, não mostrou ser um fator de risco para as RAM na admissão hospitalar. ${ }^{18}$ Este facto pode dever-se à circunstância de alguns medicamentos com risco elevado de RAM, ou margem terapêutica estreita (digoxina, insulina, anticoagulantes, quimioterapia, entre outros), não serem considerados nos critérios STOPPFrail. ${ }^{18}$

\section{Medicação potencialmente inapropriada: classes farmacológicas}

As classes farmacológicas responsáveis por cerca de um terço da MPI foram: IBP (13,26\%), antidiabéticos orais $(10,11 \%)$ e antipsicóticos $(9,89 \%)$. Num estudo que também usou a STOPPFrail verificou-se o predomínio de: IBP (22,87\%), antidislipidémicos (20,25\%), suplementos de cálcio $(14,46 \%)$ e antipsicóticos (6,61\%). ${ }^{12}$ Em estudos que usaram outros critérios e outras ferramentas foram reportadas como prevalentes as classes: antidemenciais (100\%), IBP (50\%), anti-hipertensores $(27 \%$ a $44 \%)$, antidislipidémicos $(31 \%$ a $97 \%$ ), medicina alternativa/complementar (31\%) e bifosfonatos $(27 \%) .^{44-45}$

\section{Medicamentos sem evidência clínica clara (critério}

A2)

Do total de medicamentos prescritos, 131 (11,62\%) não possuíam uma evidência clínica clara, contribuindo para $27,58 \%$ da MPI. Outros estudos mostraram que $22,8 \%$ a $43,8 \%$ dos medicamentos não tinham uma indicação clínica evidente. ${ }^{18,38,43}$ Constatou-se que os medicamentos mais prescritos, embora sem evidência clínica, eram usados no tratamento de doenças crónicas, como antianginosos, anticonvulsivantes, compostos de ferro e vasodilatadores. Assim, salienta-se a necessidade de avaliar rigorosamente a situação clínica do idoso e decidir sobre o putativo benefício da medicação crónica no FDV.

Inibidores da bomba de protões: prescrição nos últimos 12 meses de vida

Os IBP foram causa de MPI em 63 doentes (45,99\%).
No FDV não existe um consenso em relação ao uso de IBP, ${ }^{25,46}$ havendo casos de início de prescrição nesta fase da vida. ${ }^{30} \mathrm{~A}$ elevada prevalência de utilização de IBP pode estar associada ao tratamento de sintomas como dispepsia, prevenção ou tratamento de úlceras gástricas associadas à toma de anti-inflamatórios não esteroides (em sete doentes) e administração de glucocorticoides (em 15 doentes) no FDV. No contexto dos CP, os IBP podem estar indicados na prevenção e tratamento da doença de refluxo gastro-esofágico induzida por quimioterapia, no tratamento das hemoptises e na recidiva de hemorragia digestiva em doentes com história de úlcera péptica. ${ }^{47}$

\section{Antipsicóticos: prescrição nos últimos 12 meses de vida}

Os antipsicóticos foram considerados como MPI em 39 doentes $(28,47 \%)$. O seu uso nos idosos com demência é frequente, com prevalências de $27 \%$ a $30 \%$ em lares de idosos. ${ }^{48-49}$ No entanto, não existe consenso para a prescrição de antipsicóticos no FDV, havendo quem não advogue o seu uso na demência avança$\mathrm{da}^{49}$ e quem defenda que podem ser apropriados. ${ }^{25,46}$

\section{Estatinas: prescrição nos últimos 12 meses de vida}

As estatinas, para além dos IBP, representam as classes de fármacos cuja prescrição é frequentemente inapropriada no FDV, ${ }^{2,20}$ uma vez que o seu benefício demora a ser alcançado. No entanto, o diagnóstico de uma doença limitadora de vida não diminui a sua desprescrição ${ }^{50}$ com prevalências de utilização de $20,25 \%$ a 97\%.12,17,21,44,50-52 Existem, porém, recomendações sobre a não prescrição destes medicamentos em doentes com esperança de vida limitada, ${ }^{53}$ com evidência sobre a segurança da sua desprescrição, culminando na melhoria da qualidade de vida e na redução de custos em saúde. $^{54}$

\section{Vantagens e limitações do estudo}

A principal vantagem do estudo reside no conhecimento que possibilitou relativamente à prevalência da MPI e às IMED no contexto domiciliário, assim como na utilização de uma ferramenta recente, a STOPPFrail, em doentes vulneráveis.

Existem algumas limitações. O estudo foi realizado numa área geográfica restrita, o que limitou o tamanho 
da amostra, cuja seleção não foi probabilística. O estudo foi retrospetivo, pelo que os dados e as variáveis consideradas foram somente as que constavam do processo clínico dos doentes. A ausência, a não atualização ou os erros de informação dos registos podiam ter induzido um viés de informação e, consequentemente, erros de interpretação. Estes factos limitam a generalização dos resultados.

Ademais, não foram avaliados o estado funcional ou a fragilidade dos doentes, que consubstancializam aspetos fundamentais na tomada de decisão terapêutica e na análise da adequação da medicação prescrita.

Os doentes com < 65 anos foram excluídos do estudo, uma vez que tal era conveniente para aplicar os critérios STOPPFrail. Esta ferramenta não está validada para a população Portuguesa. Além disso, a STOPPFrail é recente, pelo que a comparação com outras ferramentas não pode ocorrer de modo simplista, por existirem diferenças nos critérios e nos limites de idade, não sendo valorizados alguns medicamentos de janela terapêutica estreita e responsáveis por IMED.

Algumas destas limitações poderão ser ultrapassadas com a realização de estudos prospetivos, procurando respostas não só em relação ao objetivo deste estudo, como também relativamente aos outcomes associados à MPI, como as IMED e as RAM.

\section{Implicações para o futuro}

O uso da ferramenta STOPPFrail pode ajudar os profissionais de saúde a identificar a MPI, independentemente da área de especialidade e da experiência profissional. O conhecimento sobre a MPI na população frágil é crucial para o desenvolvimento de novas orientações clínicas e para a implementação de guias de desprescrição e estratégias para reduzir a MPI. ${ }^{30}$

\section{CONCLUSÃO}

A prevalência de MPI é elevada em idosos nos últimos doze meses de vida, assim como a presença de IMED. São necessários mais estudos, de preferência multicêntricos e prospetivos, que encontrem modelos e preditores de MPI e de IMED em idosos nos últimos meses de vida.

\section{REFERÊNCIAS BIBLIOGRÁFICAS}

1. Stevenson J, Abernethy AP, Miller C, Currow DC. Managing comorbidi- ties in patients at the end of life. BMJ. 2004;329(7471):909-12.

2. Poudel A, Yates $P$, Rowett D, Nissen LM. Use of preventive medication in patients with limited life expectancy: a systematic review. J Pain Symptom Manage. 2017;53(6):1097-110.e1.

3. Boyd CM, Darer J, Boult C, Fried LP, Boult L, Wu AW. Clinical practice guidelines and quality of care for older patients with multiple comorbid diseases: implications for pay for performance. JAMA. 2005;294 (6):716-24.

4. Lindsay J, Dooley M, Martin J, Fay M, Kearney A, Barras M. Reducing potentially inappropriate medications in palliative cancer patients: evidence to support deprescribing approaches. Support Care Cancer. 2014; 22(4):1113-9.

5. Collier KS, Kimbrel JM, Protus BM. Medication appropriateness at end of life: a new tool for balancing medicine and communication for optimal outcomes - the BUILD model. Home Healthc Nurse. 2013;31(9): 518-24.

6. Riechelmann RP, Krzyzanowska MK, O'Carroll A, Zimmermann C. Symptom and medication profiles among cancer patients attending a palliative care clinic. Support Care Cancer. 2007;15(12):1407-12.

7. McNeil MJ, Hamal AH, Kutner JS, Ritchie CS, Abernethy AP. The burden of polypharmacy in patients near the end of life. J Pain Symptom Manage. 2016;51(2):178-83.e2.

8. Karuturi MS, Holmes HM, Lei X, Johnson M, Barcenas $\mathrm{CH}$, Cantor SB, et al. Potentially inappropriate medications defined by STOPP criteria in older patients with breast and colorectal cancer. J Geriatr Oncol. 2019;10(5):705-8.

9. Ma G, Downar J. Noncomfort medication use in acute care inpatients comanaged by palliative care specialists near the end of life: a cohort study. Am J Hosp Palliat Med. 2014;31(8):812-9.

10. Lees J, Chan A. Polypharmacy in elderly patients with cancer: clinical implications and management. Lancet Oncol. 2011;12(13):1249-57.

11. Lavan AH, Gallagher P, Parsons C, O'Mahony D. STOPPFrail (Screening Tool of Older Persons Prescriptions in Frail adults with limited life expectancy): consensus validation. Age Ageing. 2017;46(4):600-7.

12. Curtin D, O'Mahony D, Gallagher P. Drug consumption and futile medication prescribing in the last year of life: an observational study. Age Ageing. 2018;47(5):749-53.

13. ICD-10-CM/PCS, versão 2017/2021: tradução para Português, nova versão - 22.03.2021 [homepage]. Lisboa:Administração Central do Sistema de Saúde; 2020 [cited 2020 Ago 12]. Available from: http://www.acss.min-saude.pt/2016/07/22/portal-da-codificacao-3/

14. Infarmed. Prontuário terapêutico [homepage]. Lisboa: Infarmed; 2021 [cited 2020 Aug 12]. Available from: http://app10.infarmed.pt/prontuario/frameprimeiracapitulos.html

15. Maddison AR, Fisher J, Johnston G. Preventive medication use among persons with limited life expectancy. Prog Palliat Care. 2011;19(1):1521.

16. Sevilla-Sánchez D, Molist-Brunet N, Amblàs-Novellas J, Espaulella-Panicot J, Codina-Jané C. Potentially inappropriate medication at hospital admission in patients with palliative care needs. Int J Clin Pharm. 2017;39(5):1018-30.

17. Riechelmann RP, Krzyzanowska MK, Zimmermann C. Futile medication use in terminally ill cancer patients. Support Care Cancer. 2009;17(6): 745-8. 
18. Sevilla-Sánchez D, Molist-Brunet N, Espaulella-Panicot J, GonzálezBueno J, Solà-Bonada N, Amblàs-Novellas J, et al. Potentially inappropriate medication in palliative care patients according to STOPP-Frail criteria. Eur Geriatr Med. 2018;9:543-50.

19. West E, Costantini M, Pasman HR, Onwuteaka-Philipsen B. A comparison of drugs and procedures of care in the Italian hospice and hospital settings: the final three days of life for cancer patients. BMC Health Serv Res. 2014;14:496.

20. Todd A, Nazar H, Pearson H, Andrew L, Baker L, Husband A. Inappropriate prescribing in patients accessing specialist palliative day care services. Int J Clin Pharm. 2014;36(3):535-43.

21. Lindsay J, Dooley M, Martin J, Fay M, Kearney A, Khatun M, et al. The development and evaluation of an oncological palliative care deprescribing guideline: the 'OncPal deprescribing guideline'. Support Care Cancer. 2015;23(1):71-8.

22. Fede A, Miranda M, Antonangelo D, Trevizan L, Schaffhausser H, Hamermesz B, et al. Use of unnecessary medications by patients with advanced cancer: cross-sectional survey. Support Care Cancer. 2011;19(9): 1313-8.

23. O'Mahony D, O'Sullivan D, Byrne S, O'Connor MN, Ryan C, Gallagher P. STOPP/START criteria for potentially inappropriate prescribing in older people: version 2. Age Ageing. 2015;44(2):213-8.

24. Davies LE, Spiers G, Kingston A, Todd A, Adamson J, Hanratty B. Adverse outcomes of polypharmacy in older people: systematic review of reviews. J Am Med Dir Assoc. 2020;21(2):181-7.

25. Holmes HM, Sachs GA, Shega JW, Hougham GW, Hayley DC, Dale W. Integrating palliative medicine into the care of persons with advanced dementia: identifying appropriate medication use. J Am Geriatr Soc. 2008;56(7):1306-11.

26. Currow DC, Stevenson JP, Abernethy AP, Plummer J, Shelby-James TM. Prescribing in palliative care as death approaches. J Am Geriatr Soc. 2007;55(4):590-5.

27. Masnoon N, Shakib S, Kalisch-Ellett L, Caughey GE. What is polypharmacy? A systematic review of definitions. BMC Geriatr. 2017;17(1):230.

28. Duerden M, Avery T, Payne R. Polypharmacy and medicines optimisation: making it safe and sound [homepage]. London: The King's Fund; 2013 [cited 2020 Aug 21]. Available from: https://www.kingsfund.org. uk/publications/polypharmacy-and-medicines-optimisation

29. Morin L, Vetrano DL, Rizzuto D, Calderón-Larrañaga A, Fastbom J, Johnell K. Choosing wisely? Measuring the burden of medications in older adults near the end of life: nationwide, longitudinal cohort study. Am J Med. 2017;130(8):927-36.e9.

30. Paque K, De Schreye R, Elseviers M, Stichele RV, Pardon K, Dilles T, et al. Discontinuation of medications at the end of life: a population study in Belgium, based on linked administrative databases. Br J Clin Pharmacol. 2019;85(4):827-37.

31. McLean S, Sheehy-Skeffington B, O'Leary N, O'Gorman A. Pharmacological management of co-morbid conditions at the end of life: is less more? Ir J Med Sci. 2013;182(1):107-12.

32. Paque K, Elseviers M, Stichele RV, Pardon K, Hjermstad MJ, Kaasa S, et al. Changes in medication use in a cohort of patients with advanced cancer: the international multicentre prospective European Palliative Care Cancer Symptom study. Palliat Med. 2018;32(4):775-85.

33. Zueger PM, Holmes HM, Qato DM, Pickard AS, Calip GS, Lee TA. Use of nonpalliative medications following burdensome health care transitions in hospice patients. Med Care. 2019;57(1):13-20.

34. Todd A, Husband A, Andrew I, Pearson SA, Lindsey L, Holmes H. Inappropriate prescribing of preventative medication in patients with lifelimiting illness: a systematic review. BMJ Support Palliat Care. 2017; 7(2):113-21.

35. Poudel A, Berry R, McCarthy A, Walpole E, Yates P, Nissen L. Medication use in older, terminally ill cancer patients: are all medications appropriate? A longitudinal audit. Palliat Med. 2019;33(9):1232-5.

36. Tjia J, Velten SJ, Parsons C, Valluri S, Briesacher BA. Studies to reduce unnecessary medication use in frail older adults: a systematic review. Drugs Aging. 2013;30(5):285-307.

37. Morgan NA, Rowett D, Currow DC. Analysis of drug interactions at the end of life. BMJ Support Palliat Care. 2015;5(3):281-6.

38. Domingues D, Carneiro R, Costa I, Monteiro C, Shvetz Y, Barbosa AC, et al. Therapeutic futility in cancer patients at the time of palliative care transition: an analysis with a modified version of the Medication Appropriateness Index. Palliat Med. 2015;29(7):643-51.

39. Goh I, Lai O, Chew L. Prevalence and risk of polypharmacy among elderly cancer patients receiving chemotherapy in ambulatory oncology setting. Curr Oncol Rep. 2018;20(5):38.

40. Gaertner J, Ruberg K, Schlesiger G, Frechen S, Voltz R. Drug interactions in palliative care: it's more than cytochrome P450. Palliat Med. 2012; 26(6):813-25.

41. Riechelmann RP, Zimmermann C, Chin SN, Wang L, O'Carroll A, Zarinehbaf $S$, et al. Potential drug interactions in cancer patients receiving supportive care exclusively. J Pain Symptom Manage. 2008;35(5): 535-43.

42. Hoemme A, Barth H, Haschke M, Krähenbühl S, Strasser F, Lehner C, et al. Prognostic impact of polypharmacy and drug interactions in patients with advanced cancer. Cancer Chemother Pharmacol. 2019;83(4): 763-74.

43. Sevilla-Sanchez D, Molist-Brunet N, Amblàs-Novellas J, Roura-Poch P, Espaulella-Panicot J, Codina-Jané $C$. Adverse drug events in patients with advanced chronic conditions who have a prognosis of limited life expectancy at hospital admission. Eur J Clin Pharmacol. 2017;73(1): 79-89.

44. Oliveira L, Ferreira MO, Rola A, Magalhães M, Gonçalves JF. Deprescription in advanced cancer patients referred to palliative care. J Pain Palliat Care Pharmacother. 2016;30(3):201-5.

45. Raijmakers NJ, van Zuylen L, Furst CJ, Beccaro M, Maiorana L, Pilastri P, et al. Variation in medication use in cancer patients at the end of life: a cross-sectional analysis. Support Care Cancer. 2013;21(4):1003-11.

46. Morin L, Laroche ML, Vetrano DL, Fastbom J, Johnell K. Adequate, questionable, and inadequate drug prescribing for older adults at the end of life: a European expert consensus. Eur J Clin Pharmacol. 2018;74(10): 1333-42.

47. Reeve E, Denig P, Hilmer SN, Ter Meulen R. The ethics of deprescribing in older adults. J Bioeth Inq. 2016;13(4):581-90.

48. Tija J, Rothman MR, Kiely DK, Shaffer ML, Holmes HM, Sachs GA, et al. Daily medication use in nursing home residents with advanced dementia. J Am Geriatr Soc. 2010;58(5):880-8.

49. Toscani F, Di Giulio P, Villani D, Giunco F, Brunelli C, Gentile S, et al. Treatments and prescriptions in advanced dementia patients residing 
in long-term care institutions and at home. J Palliat Med. 2013;16(1): 31-7.

50. Silveira MJ, Kazanis AS, Shevrin MP. Statins in the last six months of life: a recognizable, life-limiting condition does not decrease their use. J Palliat Med. 2008;11(5):685-93.

51. Russell BJ, Rowett D, Abernethy AP, Currow DC. Prescribing for comorbid disease in a palliative population: Focus on the use of lipid-lowering medications. Intern Med J. 2014;44(2):177-84.

52. Matlow JN, Bronskill SE, Gruneir A, Bell CM, Stall NM, Herrmann N, et al. Use of medications of questionable benefit at the end of life in nursing home residents with advanced dementia. J Am Geriatr Soc. 2017;65(7):1535-42.

53. AMDA - The Society for Post-Acute and Long-Term Care Medicine: fifteen things physicians and patients should question. Choosing Wisely; 2013 Sep 4 [updated 2020 Nov 22; cited 2020 Aug 21]. Available from: https://www.choosingwisely.org/societies/amda-the-society-for- post-acute-and-long-term-care-medicine/

54. Kutner JS, Blatchford PJ, Taylor DH, Ritchie CS, Bull JH, Fairclough DL, et al. Safety and benefit of stopping statins in advanced life limiting disease. JAMA Intern Med. 2015;175(5):691-700.

\section{CONFLITO DE INTERESSES}

Os autores declaram não possuir quaisquer conflitos de interesse.

\section{ENDEREÇO PARA CORRESPONDÊNCIA}

Marlene Areias

E-mail: marleneareias.ter@gmail.com

https://orcid.org/0000-0002-2369-3212

Recebido em 18-11-2020

Aceite para publicação em 29-06-2021

\section{ABSTRACT}

\section{POTENTIALLY INAPPROPRIATE MEDICATION IN THE ELDERLY IN-HOME CARE IN THE LAST 12 MONTHS OF LIFE}

Introduction: Potentially inappropriate medication (PIM) is increasingly a significant subject of study due to its negative consequences in vulnerable patients.

Objective: To determine the prevalence of PIM and drug interactions in the elderly population followed by home care teams in their last twelve months of life.

Material and Methods: Quantitative, descriptive, retrospective, and observational study. Identification of PIM, using the STOPPFrail criteria, in the last twelve months of life of people with $\geq 65$ years old under follow up by community palliative care teams in the Azores islands, and who died between January 2016 and December 2018.

Results: One hundred and thirty-seven patients were included, 72 of whom were men (52.55\%), with a median age of 78 years (interquartile range $73-84)$. The prevalence of PIM was $90.51 \%(n=124)$, with frequent prescription of medication without any clear clinical evidence of $51.09 \%(n=70)$ and proton pump inhibitors of $45.99 \%(n=63)$. A total of 1,107 drug interactions were accounted for in 120 patients (87.59\%). PIM was involved in the interactions of 98 patients (71.53\%) and in 473 of the clinically significant drug interactions (46.79\%).

Conclusion: The prevalence of PIM is high in the elderly in the last 12 months of life. The use of the STOPPFrail criteria may help to identify PIM and outline a plan for its reduction, preferably in an interdisciplinary working context.

Keywords: Aged; Drug-related side effects and adverse reactions; Palliative care; Polypharmacy; Potentially inappropriate medication list. 